\title{
Lacunar thalamic stroke with pure cerebellar and proprioceptive deficits
}

\author{
José A Gutrecht, Amir A Zamani, Deepak N Pandya
}

\begin{abstract}
Case reports of two patients with cerebellar ataxia and proprioceptive sensory loss are presented. MRI of the brain revealed lesions of the ventroposterior part of the thalamus. These patients illustrate clinically the anatomical independence of cerebellar and sensory pathways in the thalamus. We suggest that the ataxic deficit is caused by interruption of cerebellar outflow pathways in the thalamus and not secondary to sensory deafferentation.
\end{abstract}

$(\mathcal{F}$ Neurol Neurosurg Psychiatry 1992;55:854-856)

Ischaemic lacunar strokes occur frequently in the thalamus. ${ }^{12}$ Small haemorrhages in the thalamus may also produce lacunar strokes ${ }^{34}$ with similar clinical deficits. ${ }^{5}$ The lateral syndrome is the most common and has one of the best known cluster of findings of the thalamic syndromes. ${ }^{6}$ This syndrome and its anatomical location of the responsible lesion were first described by Dejerine and Roussy ${ }^{7}$ in 1906.

Reported cases of "pure" thalamic syndromes with deficits in only one or two tracts or systems deficits are rare. ${ }^{8-11}$ We report two cases of lacunar thalamic stroke that produced a contralateral combination of a cerebellar deficit and proprioceptive sensory loss.

Department of

Clinic Medical Center,

Burlington,

Massachusetts

J A Gutrecht

Department of

Radiology, Brigham

and Women's Hospital,

Boston, Massachusetts

A A Zamani

Department of

Anatomy and

Neurology, Boston

University Medical

School, Boston,

Massachusetts, and

Massachusetts, and

Administration

Hospital, Bedford,

Massachusetts, USA

D N Pandya

Correspondence to:

Dr Gutrecht, Department of

Neurology, Lahey Clinic

Medical Center, 41 Mal

Road, Burlington, MA

01805.

Received 12 September

1991

and in revised form

6 December 1991.

Accepted 18 December

1991
MRI of the brain showed a small lesion in the lateral posterior aspect of the left thalamus (fig 1). No source of an embolus was found. Cerebral angiography was not performed. The deficits abated in several days.

A 74 year old right handed man complained of sudden onset of inability to control the right arm and leg despite normal strength, unsteadiness, and a "frozen feeling" in the right side of the face and right hand. History included hypertension treated with methyldopa and triamterene/hydrochlorothiazide. Mild dysarthria, inconsistently decreased pain sensation, dysdiadochokinesis, and decomposition of movements in the right limbs were noted. CT of the head showed a small left lateral thalamic haemorrhage. The findings improved in a few weeks. Neurological examination at the Lahey Clinic seven months later revealed slightly decreased touch and pain sensations but normal temperature sensation and position sense with moderately diminished two-point discrimination and graphaesthesia in the right hand. Mild dysmetria, terminal tremor, and decreased finger and foot tapping ability were present on the right side. No weakness was present, and the stretch reflexes were normoactive and symmetrical. Plantar reflexes were normal. MRI of the brain performed

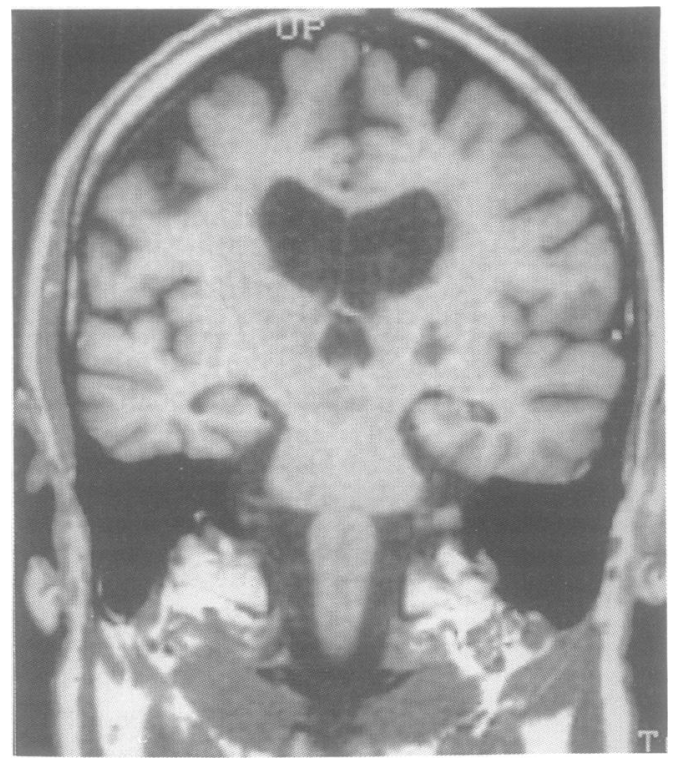

Figure 1 (Case 1). Coronal $T$,-weighted (Magneton 1.0 T, TR $0.65 \mathrm{~s}, T E 17 \mathrm{~ms}, 3 \mathrm{~mm}$ slice) image through posterior thalamic regions demonstrates a well-defined irregular area of low intensity in the lateral portion of the left thalamus. 
Figure 2 (Case 2). Coronal $T$,-weighted (Magneton 1.0 T, TR 0.65 s, TE $20 \mathrm{~ms}, 4 \mathrm{~mm}$ slice) image through posterior thalamic regions demonstrates a linear area of low signal intensity in the lateral portion of the the lateral portio

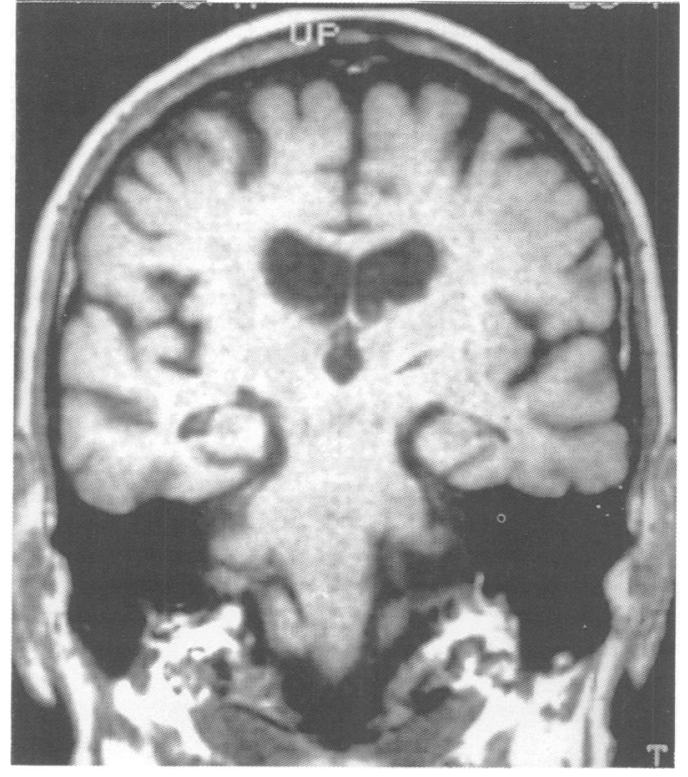

almost one year later showed a lesion in the lateral posterior area of the left thalamus (fig 2).

\section{Discussion}

Thalamic ischaemic strokes frequently produce multiple symptoms and signs because of involvement of different nuclei as well as subthalamic regions that share blood supply. Small haemorrhages in these areas may produce similar symptoms. ${ }^{5}$ The best known and most common thalamic syndrome is the classic lateral or Dejerine-Roussy ${ }^{7}$ syndrome. On the other hand, well-defined "pure" thalamic syndromes are uncommon. These "pure" syndromes are characterised by involvement of one or two tracts. Isolated proprioceptive sensory loss, ${ }^{8}$ pure sensory deficit, ${ }^{9}$ ataxic hemiparesis, ${ }^{10}$ and cerebellar syndromes ${ }^{11}$ have been reported.

Our two patients had deficits in the proprioceptive and more impressively, cerebellar domains. The cerebellar deficits were typical and did not worsen with closure of the eyes. Since the original description by Dejerine and Roussy, ${ }^{7}$ sensory deficits have been ascribed to a lesion in the ventral posterior nucleus. The origin of hemiataxia in these patients, however, has caused some argument. In 1908, Vincent ${ }^{12}$ was the first to suggest that the cerebellar deficit was caused by involvement of cerebellar pathways in the subthalamic region as they enter the thalamus, although no pathological confirmation was available. Dejerine and Roussy, ${ }^{7}$ however, believed that the ataxic component in the syndrome was from the sensory deficits. In 1955, Garcin $^{13}$ presented the case of a patient who had increasing hemiataxia while severe deficits of proprioceptive sensation in the same limbs subsided. Anatomical examination of the brain revealed a lacuna in the posterolateral region of the contralateral thalamus. $\mathrm{He}^{13}$ stated that the cerebellar deficits were explained by the thalamic lesion without subthalamic involvement.
Lack of causal correlation of sensory and cerebellar deficits was further suggested by the case reported by Garcin and Lapresle ${ }^{11}$ in 1969. Their patient had no sensory deficits, and the lacuna found at necropsy was on the medial aspect of the contralateral thalamus. In the series of Bogousslavsky et al ${ }^{14}$ five of 18 patients with the lateral thalamic syndrome had severe ataxia "unrelated" to sensory deficits. Other cases of sensory deficits without ataxia have been reported, ${ }^{5}$ thus strongly suggesting independence of ataxic limb movement and sensory deficits. The patient described by Boiten and Lodder ${ }^{10}$ had cerebellar ataxia without sensory deficits. Sacco et al ${ }^{9}$ suggested that the limb ataxia in one patient with "pure" proprioceptive sensory loss was caused by the sensory deficits although the ataxic movements were present only in the arm and not in the leg despite sensory loss in both limbs. Recently, Dobato et $a l^{15}$ suggested that the incoordination of limb movements was caused by sensory deafferentation rather than involvement of cerebellar pathways.

Anatomical studies ${ }^{16}$ in monkeys have shown that dense input of cerebellar fibres to the contralateral thalamus exists. These fibres arise in the dentate nucleus of the cerebellum and terminate in the cell-sparse ventral posterolateral nucleus, oral division and ventral lateral nucleus, caudal division of the thalamus whereas the posterior columns medial lemniscal fibres terminate in the adjacent ventral posterolateral caudalis nucleus. These two tracts do not overlap in the thalamus. However, some overlap of cerebellar and spinothalamic fibres occurs as some of the latter terminate in the ventral posterolateral nucleus, oral division. ${ }^{16}$ It is therefore conceivable that a small lesion in the ventroposterior area of the thalamus could produce deficits in sensory or cerebellar pathways or both according to its location. The size and location of the lacunar lesion would be defined by the territory of the blood supply and size of the terminal perforating branches of the thalamogeniculate arterial stalk or haemorrhage.

We suggest that the hemiataxia in our two patients is independent of the sensory deficits and is caused by involvement of cerebellar pathways in the thalamus. The independence of the cerebellar and proprioceptive sensation systems agrees with the known neuroanatomy and functional connections of the thalamus. ${ }^{17}$

1 Fisher CM. Lacunes: Small, deep cerebral infarcts. Neurology 1965;15:774-84.

2 Orgogozo JM, Bogousslavsky J. Lacunar syndromes. In: Toole JF, ed. Handbook of clinical neurology. Vascular diseases Part II. New York: Elsevier, 1989:235-69.

3 Mori E, Tabuchi M, Yamadori A. Lacunar syndrome due to intracerebral hemorrhage. Stroke 1985;16:454-9.

4 Millikan C, Futrell N. The fallacy of the lacune hypothesis. Stroke 1990;21:1251-7.

5 Kawahara N, Sato K, Muraki M, Tanaka K, Kaneko M, Uemura $K$. CT classification of small thalamic hemorthages and their clinical implications. Neurology 1986;36:165-72.

6 Martin J. Thalamic syndromes. In: Vinken PJ, Bruyn GW, eds, Handbook of clinical neurology. Amsterdam: NorthHolland, 1968:469-96.

7 Dejerine J, Roussy G. Le syndrome thalamique (1). Rev Neurol 1906;12:521-32.

8 Fisher CM. Pure sensory stroke involving face, arm, and leg. Neurology 1965;15:76-80. 
9 Sacco RL, Bello JA, Traub R, Brust JCM. Selective proprioceptive loss from a thalamic lacunar stroke. Stroke 1987;18:1160-3.

10 Boiten J, Lodder J. Ataxic hemiparesis following thalamic infarction. Stroke 1990;21:339-40.

11 Garcin R, Lapresle J. Incoordination cérébelleuse du membre inférieur par lésion localisée dans la région interne du thalamus contro-latéral. Rev Neurol 1969; 120:5-13.

12 Vincent C. Syndrome thalamique avec troubles cérébelleux et vaso-asymétrie. Rev Neurol 1908;16:553-6.

13 Garcin R. Syndrome cérébello-thalamique par lésion localisée du thalamus-Avec une digression sur le "signes de la main creuse" et son intérêt séméiologique. Rev Neurol
14 Bogousslavsky J, Regli F, Uske A. Thalamic infarcts: Clinical syndromes, etiology, and prognosis. Neurology 1988;38:837-48.

15 Dobato JL, Villanueva JA, Giménez-Roldán S. Sensory ataxic hemiparesis in thalamic hemorrhage. Stroke 1990;21:1749-53.

16 Asanuma C, Thach WT, Jones EG. Distribution of cerebellar terminations and their relation to other afferent terminations in the ventral lateral thalamic region of the monkey. Brain Res Rev 1983;5:237-65.

17 Ilinsky IA, Kultas-Ilinsky K. Sagittal cytoarchitectonic maps of the Macaca mulatta thalamus with a revised nomenclature of the motor-related nuclei validated by observations on their connectivity. $f$ Comp Neurol 1987; 262:331-64. 